\title{
Aproximación Diagnóstica De Las Enfermedades Sistémicas De Los Pacientes Con Signos Y Lesiones Oculares Atendidos En La Consulta Externa Del Hospital Veterinario De Animales De Compañía De La Universidad De San Carlos De Guatemala
}

\author{
By Laparra-Galindo, Julia Patricia \& Mendez-Valenzuela Carlos Roberto
}

Abstract- The goal of this article is to show the most frequent types of differential diagnoses of systemic diseases in canines with ocular signs and lesions. For this purpose, a retrospective study was conducted on 510 medical records from patients of the Companion Animal Veterinary Hospital (HVAC) of the University of San Carlos (USAC) of Guatemala, from August to November 2017. The inclusion criteria were: canine species, having a differential diagnosis established list of possible systemic diseases and an eye examination by specialist. Through a descriptive analysis of the data, it was established that, of the 510 canines examined, 212 (42\%) presented signs and ocular alterations, of these there were 550 differential diagnosis, which were categorized using the acronym DAMNIT $\vee$; from which it was determined that $40 \%$ are infectious / inflammatory, neoplasic (19\%) followed by metabolic processes (13\%), allergic / autoimmune (10\%), degenerative (7\%), idiopathic (5\%), traumatic / toxic $4 \%$ and finally vascular (2\%).

Keywords: canine, DAMNIT V, differential diagnosis, veterinary ophthalmology.

GJMR-G Classification: NLMC Code: WA 360

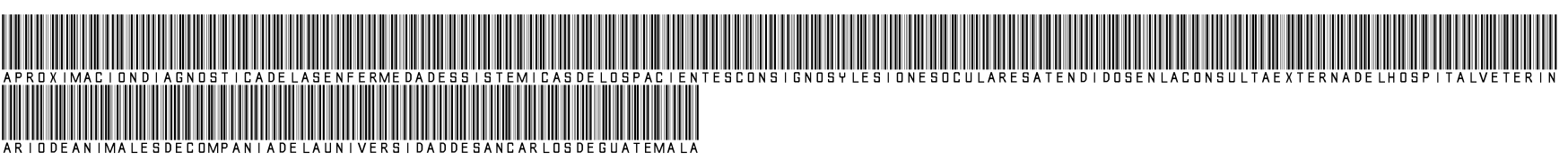

Strictly as per the compliance and regulations of:

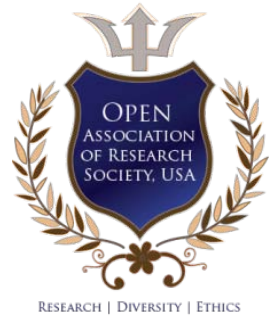

(c) 2021. Laparra-Galindo, Julia Patricia \& Mendez-Valenzuela Carlos Roberto. This research/review article is distributed under the terms of the Attribution-NonCommercial-NoDerivatives 4.0 International (CC BY-NC-ND 4.0). You must give appropriate credit to authors and reference this article if parts of the article are reproduced in any manner. Applicable licensing terms are at https://creativecommons.org/licenses/by-nc-nd/4.0/. 


\title{
Aproximación Diagnóstica De Las Enfermedades Sistémicas De Los Pacientes Con Signos Y Lesiones Oculares Atendidos En La Consulta Externa Del Hospital Veterinario De Animales De Compañía De La Universidad De San Carlos De Guatemala
}

\author{
Laparra-Galindo, Julia Patricia ${ }^{\alpha} \&$ Mendez-Valenzuela Carlos Roberto ${ }^{\sigma}$
}

Resumen- El objetivo de este trabajo es evidenciar los tipos de diagnósticos diferenciales de enfermedades sistémicas más frecuentes en caninos con signos y lesiones oculares. Para ello se realizó un estudio retrospectivo en 510 registros médicos de pacientes de la consulta externa del Hospital Veterinario de Animales de Compañía (HVAC) de la Universidad de San Carlos (USAC) de Guatemala, correspondientes al período de agosto a noviembre de 2017. Los criterios de inclusión fueron: pertenecer a la especie canina, tener una lista establecida de diagnósticos diferenciales de sus posibles patologías sistémicas y evaluación oftalmológica por especialista. A través de un análisis descriptivo de los datos se estableció que, de los 510 caninos evaluados, 212 (42\%) presentaron signos y alteraciones oculares, de estos se totalizaron 550 diagnósticos diferenciales, los cuales fueron categorizados utilizando el acrónimo VITAMIND; de donde se determinó que el $40 \%$ son de tipo infeccioso/inflamatorio, neoplásicos (19\%) seguidos de procesos metabólicos (13\%), alérgicos/autoinmunes (10\%), degenerativos (7\%), idiopáticos (5\%), traumáticos/tóxicos $4 \%$ y por último vasculares (2\%).

Debido a una tendencia de obviar la enfermedad ocular, la importancia de este artículo radica en informar al clínico sobre la relación de las alteraciones oculares con procesos sistémicos, ya que la identificación de lesiones oftálmicas nos puede orientar al diagnóstico y monitorización de las enfermedades sistémicas y al mismo tiempo tratar los efectos secundarios que estas puedan tener a nivel ocular.

Palabras clave: canino, diagnóstico diferencial, oftalmología veterinaria, VITAMIND.

Abstract- The goal of this article is to show the most frequent types of differential diagnoses of systemic diseases in canines with ocular signs and lesions. For this purpose, a retrospective study was conducted on 510 medical records from patients of the Companion Animal Veterinary Hospital (HVAC) of the University of San Carlos (USAC) of Guatemala, from August to November 2017. The inclusion criteria were: canine species, having a differential diagnosis established list of possible systemic diseases and an eye examination by specialist. Through a descriptive analysis of the data, it was established that, of the 510 canines examined, 212 (42\%) presented signs and ocular alterations, of these there were 550 differential diagnosis, which were categorized using the acronym

Author a: e-mail: dra.patricialaparra@gmail.com

Author o: e-mail: cmendezv@purdue.edu
DAMNIT V; from which it was determined that $40 \%$ are infectious / inflammatory, neoplasic (19\%) followed by metabolic processes (13\%), allergic / autoimmune (10\%), degenerative $(7 \%)$, idiopathic $(5 \%)$, traumatic / toxic $4 \%$ and finally vascular $(2 \%)$.

Due to a tendency to obviate ocular disease, the purpose of this article is to broaden the clinician's criteria to relate ocular alterations with systemic processes, since the identification of ocular manifestations can guide them in the diagnosis and monitoring of systemic diseases and at the same time treat the side effects that these can have at the ocular level.

Keywords: canine, DAMNIT V, differential diagnosis, veterinary ophthalmology.

\section{INTRODUCCIÓN}

- I ojo es un órgano muy complejo en términos de estructura y función. Además, es un órgano muy sensible, cuya función puede verse afectada con un leve insulto a su homeostasis debido a una lesión directa o a enfermedades locales y sistémicas (Kurup, Parikh, Mahla y Ratnu, 2017). La importancia de estas lesiones tiene relación con dos circunstancias: La primera es que la extensión de la patología sistémica puede provocar signos oftalmológicos. "La segunda circunstancia que hemos de considerar es que en ocasiones los signos oculares pueden ser la primera manifestación de alguna enfermedad sistémica y su aparición por lo tanto nos puede conducir al diagnóstico". En cualquiera de las circunstancias el hallazgo precoz de las lesiones oculares nos ayudara a implementar un tratamiento temprano para evitar lesiones irreversibles (Andonegui, 2008).

Otro aporte importante que nos da el examen ocular es que en ninguna otra parte del cuerpo se puede observar directamente el sistema circulatorio o examinar el tejido nervioso sin la necesidad de hacer una biopsia invasiva. Es por ello por lo que la valoración oftalmológica es de suma importancia como complemento del examen físico ya que nos permite el diagnóstico de alteraciones oculares y también el de enfermedades sistémicas que pueden haber pasado 
desapercibidas en el examen físico general (Ettinger y Feldman, 2017).

Además de lo anterior, las enfermedades cerebrovasculares cursan con diferentes expresiones clínicas neuro-oftalmológicas. Esto debido a que los vasos retinianos comparten características anatómicas, fisiológicas y embriológicas con los vasos cerebrales. La retina es una extensión del diencéfalo y posee una barrera hemato-retiniana similar a la hemato-encefálica (Gelatt y Plummer, 2017). "Los cambios en los vasos retinianos reflejan probablemente cambios en los vasos cerebrales. Sin embargo, la irrigación retiniana es la única que puede ser visualizada en vivo y de forma directa sin utilizar medidas o medios invasivos". Es por esto por lo que signos vasculares retinianos pueden darnos claves muy importantes para comprender las enfermedades cerebrovasculares relacionadas (ZabidiHussin, 2016). Estas, entre otras patologías de origen sistémico, representan una amenaza para la integridad funcional del órgano visual. Es por ello por lo que es importante que el médico esté familiarizado con el espectro de la afectación ocular en las enfermedades sistémicas, ya que la intervención adecuada y la derivación puede evitar secuelas importantes e incluso la ceguera del paciente. (Lenake y Du Toit, 2014)

De acuerdo con Andrade (2008) el veterinario general tiene conocimientos de anatomía y fisiología ocular para realizar un examen completo del ojo, pero se necesita una capacitación técnica para lograr un examen oftalmológico más completo y de calidad, especialmente con respecto al manejo del equipo específico que se requiere. Con el crecimiento de las especialidades en medicina veterinaria, herramientas tales como el oftalmoscopio se incluyen entre los principales utensilios del oftalmólogo veterinario, pero todavía son poco conocidos por los médicos veterinarios generales (Freitas, Montiani-Ferreira, y Ferreira, 2018).

La lista de diagnósticos diferenciales es uno de los aspectos más importantes para la aproximación diagnóstica. Esta debe de contemplar cada uno de los problemas que presente un paciente en la historia clínica de la enfermedad, exámen físico y pruebas diagnósticas (Gough, 2007). El acrónimo VITAMIND (V= vascular, $\quad I=\quad$ infeccioso/inflamatorio, $\quad T=$ traumático/tóxico, $\mathrm{A}=$ autoinmunes, $\mathrm{M}=$ metabólicas, $\mathrm{I}=$ idiopáticas, $\mathrm{N}=$ neoplásicas/nerviosas, $\mathrm{D}=$ degenerativas) es una herramienta con categorías amplias de diagnósticos diferenciales utilizado como recordatorio de que cada problema tiene una multitud de causas potenciales que deben de considerarse hasta ser descartadas. (Englar, 2019) El examen ocular es un componente esencial que puede reducir esta lista de probables diagnósticos diferenciales en los pacientes con enfermedades sistémicas (Cullen y Webb, 2013).

\section{il. Materiales y Métodos}

Se realizó un estudio retrospectivo de 510 registros médicos correspondientes al período de agosto a noviembre del 2017 de pacientes que se presentaron a la consulta externa del HVAC de la USAC de Guatemala, ubicada en la zona 12 de la Ciudad de Guatemala. Los criterios de inclusión fueron: ser de especie canina, tener una lista establecida de diagnósticos diferenciales de su patología sistémica y poseer una evaluación ocular por especialista. El equipo utilizado para la evaluación oftalmológica fue una lámpara de hendidura, oftalmoscopio Panoptic, tonómetro neumático, lente de Goldman y una lente de 20 Dioptrías. Los insumos y medicamentos utilizados fueron test de Schirmmer, tinciones de fluoresceína, rosa de bengala, anestésico local y midriáticos. Las evaluaciones oftalmológicas se realizaron con el paciente despierto.

Del total de registros médicos analizados, fueron seleccionados 212 correspondientes a pacientes caninos con enfermedades sistémicas que cumplían los criterios de inclusión. De cada registro médico se recopiló la lista establecida de diagnósticos diferenciales de cada paciente. Posteriormente se utilizó el acrónimo VITAMIND para categorizar los diferentes diagnósticos diferenciales. Para el análisis de los datos se utilizó estadística descriptiva utilizando distribuciones de frecuencias para las variables.

\section{ili. Resultados}

Se evaluaron un total de 510 registros médicos de pacientes atendidos en la consulta externa del HVAC de agosto a noviembre del 2017 y se determino que el $42 \%$ presentaban signos y lesiones oftalmológicas.

Tabla 1: Número de pacientes que presentaron signos y lesiones oculares

\begin{tabular}{|ccc|}
\hline Tipo de paciente & Pacientes & $\%$ \\
\hline $\begin{array}{c}\text { Con signos y Lesiones } \\
\text { Oftalmológicas }\end{array}$ & 212 & $42 \%$ \\
Sin Signos y Lesiones Oftalmológicas & 298 & $58 \%$ \\
\hline Total & 510 & $100 \%$ \\
\hline
\end{tabular}


Tabla 2: Categoría de los diagnósticos diferenciales

\begin{tabular}{|lcc|}
\hline \multicolumn{1}{c}{ Categoría } & $\begin{array}{c}\text { Diagnósticos } \\
\text { diferenciales }\end{array}$ & $\%$ \\
\hline Vasculares & 13 & $2 \%$ \\
Infecciosos/Inflamatorios & 222 & $40 \%$ \\
Traumáticos/Tóxicos & 20 & $4 \%$ \\
Autoinmunes & 54 & $10 \%$ \\
Metabólicos & 71 & $13 \%$ \\
Idiopáticos & 26 & $5 \%$ \\
Neoplásicos/Neurológicos & 104 & $19 \%$ \\
Degenerativos & 40 & $7 \%$ \\
\hline \multicolumn{1}{|c}{ Total } & 550 & $100 \%$ \\
\hline
\end{tabular}

\section{Discusión de Resultados}

En este estudio se determinó que el examen ocular es un complemento importante del proceso del examen físico; ya que se reconocieron alteraciones oftalmológicas en el $42 \%$ de los pacientes caninos que se presentaron a la consulta general del HVAC. Porcentaje muy diferente al que reportan estudios realizados en clínicas veterinarias generales como el de Manizales, Colombia, en el que se concluyó que de los pacientes atendidos solo al $4.56 \%$ se les detecto afección oftalmológica (Muñoz, Ramírez, y Garcés, 2015). Porcentaje muy similar fue reportado por el sistema de vigilancia veterinaria de animales de compañía del Reino Unido con un 4.9\%. (The People's Dispensary for Sick Animals, 2019). Esta diferencia de porcentajes puede atribuirse a que los médicos veterinarios generales tienden a considerar al ojo y sus anexos como estructuras diferentes al resto de los tejidos no oculares. Esto conduce a la omisión del examen oftálmico en pacientes con enfermedades sistémicas generales. Efectivamente, el ojo es un órgano con anatomía y fisiología muy similar a las de otros órganos y tejidos, pero se requiere de un entrenamiento y equipo especial para su exploración (Ettinger y Feldman, 2017). Mitchell (2011), también afirma que los exámenes oculares requieren equipo básico, buena observación clínica, mucha práctica y que además es importante que los clínicos estén familiarizados con la anatomía y fisiología normal del ojo, para que se puedan reconocer las anomalías y apreciar la importancia de las alteraciones oculares.

Otro hallazgo importante es que el $40 \%$ de los diagnósticos diferenciales establecidos para las patologías sistémicas de los caninos con signos y lesiones oculares fueron de tipo infeccioso-inflamatorio. Datos similares fueron encontrados en un estudio prospectivo de enfermedades oculares y perioculares en animales en Minas, Brasil, donde por medio de necropsia y examen a otros órganos, se concluyó que numerosos animales que presentaron lesiones oculares tenían enfermedades sistémicas de tipo infeccioso- inflamatorio (Moreira, Neto, Langohr, y Ecco, 2018). Un dato importante que hay que considerar en este grupo es que las enfermedades de tipo infeccioso, como en el caso del distemper canino, los signos oculares son las primeras manifestaciones de la enfermedad sistémica, presentándose lesiones fáciles de identificar como conjuntivitis aguda de leve a grave, bilateral, serosa a mucopurulenta y principalmente con afectación de la conjuntiva palpebral. Cuando la enfermedad ha avanzado causando trombocitopenia y vasculitis, los signos oculares son más complejos e incluyen uveítis hemorrágica, hifema y hemorragias retinianas que provocan desprendimiento de retina y neuritis óptica (Aroch, Ofri, y Sutton, 2008). Y aunque en este grupo de pacientes la etiología de lesiones oculares secundarias a procesos infecciosos-inflamatorios puede ser diversa, Massa, Gilger, Miller y Davidson (2002), demostraron que el 17.6\% de la inflamación del tracto uveal es debida a causas infecciosas siendo el agente causal más común en perros la Ehrlichia canis. La uveítis no es solo un signo ocular importante en perros infectados con E. canis, pues se reportan, además, casos de desprendimiento de retina, conjuntivitis, petequias y equimosis en iris, edema de córnea, glaucoma secundario, neuritis óptica, hifema y hemorragias (Trujillo D., Quijano J., Padilla B. y Sánchez M., 2019).

El segundo grupo de diagnósticos diferenciales de enfermedades sistémicas con signos y lesiones oculares más frecuente con el $19 \%$ es de tipo neoplásico - nervioso. Este resultado es porque los pacientes con neoplasias sistémicas pueden cursar con procesos oculares inflamatorios secundarios o debido a metástasis intraoculares como documentan Conceicão, Ribeiro, Piso, y Laus, (2010), quienes indican que el linfoma en perros es la metástasis intraocular más común presentándose en el 37\% y se manifiesta como anomalías en el segmento anterior, uveítis moderada a grave, hemorragias, hipopión, infiltración corneal, desprendimiento de retina y hemorragias retinianas. También los adenocarcinomas de tejido mamario, tiroides, hígado, páncreas y útero 
son frecuentes. Otras neoplasias secundarias para considerar en este grupo son los condrosarcomas, hemangiosarcomas, melanomas cutáneos y orales, carcinoma de las células transicionales de la vejiga urinaria, sarcomas neurogénicos, seminomas, osteosarcomas, rabdomiosarcomas entre otras.

En cuanto a los pacientes con diferenciales de enfermedad sistémica nerviosa, el HVAC cuenta con una ficha en la cual se incluyen los signos y lesiones neuro oftalmológicas. Estos se pueden agrupar en cuatro categorías: 1. trastornos de la visión (ceguera central / pérdida parcial de la visión), 2. trastornos del tamaño y función pupilar (miosis, midriasis, anisocoria, Síndrome de Horner, 3. trastornos de la posición del globo ocular (estrabismo) 4. movimientos oculares involuntarios (nistagmo), (Brisimi, 2018). Estos signos son de importancia para la neurolocalización de una lesión, como por ejemplo el nistagmo, que al igual que en la medicina humana, podría sugerir una localización neuroanatómica cerebelosa (Ives, Mackillo y Olby, 2018). También hay otras alteraciones oftalmológicas en las que el sistema nervioso central puede estar involucrado como son los trastornos del párpado (ptosis), protrusión del tercer párpado y epífora. En sí, el conocimiento de la neuroanatomía y la localización precisa de las lesiones optimizaría los diagnósticos diferenciales para llegar a un diagnóstico final y tratamiento adecuado. (Brisimi, et al 2018)

Un grupo no menos importante es el de pacientes con alteraciones oftalmológicas y diagnósticos diferenciales de tipo metabólico. En este estudio se identificaron lesiones oculares en el $13 \%$ de los pacientes con diferenciales de enfermedad metabólica. Esto puede ser debido a que, según López S, Laparra J, Chávez, Villatoro D, (2019), el hipotiroidismo y la obesidad resultaron ser los desórdenes metabólicos más frecuentes en el HVAC de la USAC Guatemala y los pacientes con endocrinopatías desarrollan diversas afecciones oftálmicas como resultado de los desequilibrios hormonales. Por ejemplo, los animales diabéticos desarrollan cataratas, uveítis, queratopatías, retinopatías y al igual que los pacientes con Cushing, desarrollan lesiones oculares secundarias a hipertensión sistémica y a los trastornos en el metabolismo de los lípidos. (Plummer, Specht, y Gelatt, 2007). Otra causa de la pérdida de la transparencia corneal en este tipo de patologías sistémicas es por el acúmulo de calcio o su combinación con lípidos que provocan degeneración corneal (Zapata, Mv, Pn, y Gl, n.d.). Se debe de tener en cuenta que en todas las especies existen posibles manifestaciones oculares cuando se elevan las lipoproteínas circulantes. (Crispin, 2002). Meana y colaboradores sostienen que el diagnóstico de degeneración corneal en el canino amerita un examen semiológico completo del paciente para diagnosticar enfermedades sistémicas que alteren la lipemia e instaurar un tratamiento para mantener la normolipemia y por ende la transparencia corneal (Zapata et al., n.d.).

Según los resultados de este estudio los procesos de carácter autoinmune tuvieron un 10\% de los pacientes que presentaron alteraciones oculares. Esto debido a que las enfermedades de tipo inmunológico que ocurren a nivel oftálmico son similares a las del resto del organismo. Los procesos inmunológicos pueden llegar a afectar el tracto uveal, siendo esta la etiología de una serie de inflamaciones del globo ocular que pueden resultar en secuelas permanentes. Estas inflamaciones requieren especial distinción de otras patologías oculares locales que producen ojo rojo. (Ettinger y Feldman, 2017)

Abreviaturas:

HVAC: Hospital Veterinario de la Facultad de Medicina Veterinaria y Zootecnia

USAC: Universidad de San Carlos

\section{References Références Referencias}

1. Andonegui, J. (2008). Manifestaciones oculares de las enfermedades sistémicas. Anales Del Sistema Sanitario de Navarra.

2. Andrade, A. (2008). Semiología do Sistema Visual dos Animais Domésticos. Semiología Veterinaria, Sao Pablo, Cap. 14, p. 623-653.

3. Aroch, I., Ofri, R., \& Sutton, G. A. (2008). Ocular Manifestations of Systemic Diseases. Slatter's Fundamentals of Veterinary Ophthalmology, 374. https://doi.org/10.1016/B978-072160561-6.50021-6

4. Brisimi N., Liatis Th., Komnenou A., Charalambidou G., Soubasis N. y Polizopoulou Z., (2018). Neuroophthalmological abnormalities in neurological diseases of dogs and cats: a retrospective study of 114 cases (2010-2015). Journal of the Hellenic Veterinary Medical Society, 68(3), 433-444. doi: https://doi.org/10.12681/jhvms.15538

5. Conceicão, L. F. da, Ribeiro, A. P., Piso, D. Y. T., \& Laus, J. L. (2010). Considerations about ocular neoplasia of dogs and cats. Ciência Rural, 40(10), 2235-2242. https://doi.org/10.1590/S0103-847820 10005000165

6. Crispin, S. (2002). Ocular lipid deposition and hyperlipoproteinemia. Progress in Retinal and Eye Research. https://doi.org/10.1016/S1350-9462(02) 00004-6

7. Cullen, C. L., \& Webb, A. (2013). Ocular manifestations of systemic disease. Part 1: The Dog. In Veterinary Ophthalmology.

8. Gough A., 2007. Diagnosis in Small Animal Medicine, Blakwell Publishing, USA, first edition, 453 p.p.

9. Englar, R. E. (2019). The Problem-Oriented Approach to Clinical Medicine. Common Clinical 
Presentations in Dogs and Cats, 1-10. https://doi.org/10.1002/9781119414612.CH1

10. Ettinger, S. J., \& Feldman, E. C. (2017). Textbook of veterinary internal medicine: diseases of the dog and the cat. Elsevier.

11. Freitas, H. D. M., Montiani-Ferreira, F., \& Ferreira, T. A. C. (2018). A HISTÓRIA DA OFTALMOSCOPIA NA CIÊNCIA MÉDICA E VETERINÁRIA. Archives of Veterinary Science. https://doi.org/10.5380/avs. v23i4.57689

12. Gough A., 2007. Diagnosis in Small Animal Medicine, Blakwell Publishing, USA, first edition, 453 p.p.

13. Ives, E., MacKillop, E y Olby N., (2018). Saccadic oscillations in 4 dogs and 1 cat. Journal of Veterinary Internal Medicine, 32(4), 1392-1396. https://doi.org/10.1111/JVIM.15144

14. Lenake, M., \& Du Toit, N. (2014). The eye in systemic disease. South African Family Practice. https://doi.org/10.1080/20786204.2014.10844577

15. Massa K., Gilger B., Miller T, and Davidson M., (2002). Causes of uveitis in dogs: 102 cases (19892000), American College of Veterinary Ophthalmologists, Veterinary Ophthalmology, 5, 93-98.

16. Meana M., Cassagne P. y Zapata G., 2011, Corneal degeneration in a canine as only sign of hypothyroidism, report of a case, Anacleta Vet, 31 (2): 33- 36.

17. Moreira, M., Teixeira R., Langohr, I., y Ecco, R. (2018). Prospective study of ocular and periocular diseases in animals: 188 cases. Pesquisa Veterinaria Brasileira, 38(3), 502-510. https://doi.org/10.1590/1678-5150-PVB-5044

18. Muñoz, J., Ramírez, G. F., \& Garcés, L. F. (2015). Consulta externa en clínicas veterinarias de Manizales. Análisis epidemiológico en Caninos. Spei Domus, 11(23). https://doi.org/10.16925/ sp.v11i23.1364

19. Plummer, C., Specht, A., \& Gelatt, K. (2007). Ocular manifestations of endocrine disease. Compendium Continuing Education for the Practising Veterinarian. https://doi.org/10.1016/B978-1-56053-442-6.50036$X$

20. López, S., Laparra, J., Chávez, J., Villatoro, D., (2019). Caracterización De Pacientes Caninos De Raza Pura Con Diagnostico De Enfermedad Metabólica Atendidos En El Hospital Veterinario De La Universidad De San Carlos De Guatemala. Global Journal of Medical Research, 19(2), 33-39. https://doi.org/10.34257/gjmrgvol19is2pg33

21. The People's Dispensary for Sick Animals, 2019. PDSA ANIMAL WELLBEING (PAW) REPORT 2019. Retrieved May 1, 2021, from https://www.pdsa.org. uk/media/7420/2019-paw-report_downloadable.pdf

22. Trujillo D., Quijano J., Padilla B. y Sánchez M., (2019). Hallazgos oculares como factores predictivos y diagnósticos de ehrlichiosis canina. Revista de Investigaciones Veterinarias del Perú, 30, (3), 1226-1233.

23. Zapata, G., Mv, M., Pn, C., \& Gl, Z. (n.d.). Degeneración corneal canina 33 Impresa Dirección para correspondencia: Corneal degeneration in a canine as only sign of hypothyroidism, report a case. 